\title{
Aqueous Level of ANGPTL4 Correlates with the OCTA Metrics of Diabetic Macular Edema in NPDR
}

\author{
Qing Xu, Chaoju Gong, Lei Qiao, Ruifang Feng, Haiyang Liu, Yalu Liu, Sujuan Ji, \\ Yipeng Zhang, Shuang Wu, and Suyan $\mathrm{Li}$ i
}

\author{
Department of Ophthalmology, The Affiliated Xuzhou Municipal Hospital of Xuzhou Medical University, Xuzhou First \\ People's Hospital, Xuzhou Eye Disease Prevention and Treatment Institute, Xuzhou, 221116 Jiangsu Province, China \\ Correspondence should be addressed to Suyan Li; lisuyan_med@163.com
}

Received 30 August 2021; Revised 9 November 2021; Accepted 18 December 2021; Published 19 January 2022

Academic Editor: Lei Liu

Copyright (c) 2022 Qing Xu et al. This is an open access article distributed under the Creative Commons Attribution License, which permits unrestricted use, distribution, and reproduction in any medium, provided the original work is properly cited.

\begin{abstract}
Purpose. To investigate the aqueous levels of angiogenic factors in nonproliferative diabetic retinopathy (NPDR) patients with diabetic macular edema (DME) and to ascertain their association with optical coherence tomography angiography (OCTA) metrics. Methods. This study enrolled 21 NPDR eyes with DME (NPDR/DME+), 17 NPDR eyes without DME (NPDR/DME-), and 16 diabetic eyes without retinopathy (DWR). Luminex bead-based multiplex array was used to measure the levels of 25 cytokines. OCTA system with a scan area of $3 \times 3 \mathrm{~mm}$ was used to measure retinal thickness (RT), retinal volume (RV), superficial vessel density (SVD), deep vessel density (DVD), foveal avascular zone (FAZ) area, perimeter and acircularity index. Results. The levels of ANGPTL4 were significantly different among the three groups $(P<0.05)$, in which NPDR/DME+ group had the highest level and NPDR/DME- group had a higher level than the DWR group (all, $P<0.0167)$. OCTA examination showed that, compared with DWR and NPDR/DME- group, RT and RV increased and the whole/parafoveal DVD decreased in NPDR/DME+ group (all, $P<0.05$ ). Meanwhile, NPDR/DME- group had lower parafoveal DVD than the DWR group $(P<0.05)$. Correlation analysis showed that the levels of ANGPTL4 were positively correlated with foveal/parafoveal RT and RV and negatively correlated with whole/parafoveal DVD in NPDR patients (all, $P<0.05$ ). As the influencing factor of RT, $\mathrm{RV}$, and DVD, every additional $10^{3} \mathrm{pg} / \mathrm{ml}$ of ANGPTL4 was associated with an increase in foveal and parafoveal RT of $4.299 \mu \mathrm{m}$ and $3.598 \mu \mathrm{m}$, respectively. Every additional $10^{6} \mathrm{pg} / \mathrm{ml}$ of ANGPTL4 was associated with an increase in foveal and parafoveal RV of $3.371 \mathrm{~mm}^{3}$ and $17.705 \mathrm{~mm}^{3}$, respectively. Every additional $10^{4} \mathrm{pg} / \mathrm{ml}$ of ANGPTL4 was associated with a decrease in whole and parafoveal DVD of $1.705 \%$ and $1.799 \%$, respectively. Conclusions. The level of ANGPTL4 in aqueous humor of NPDR patients with DME was significantly increased and ANGPTL4 might predict RT, RV, and parafoveal DVD of DME in NPDR patients.
\end{abstract}

\section{Introduction}

Diabetic retinopathy (DR) is one of the most destructive microvascular complications of diabetes mellitus. Intraocular neovascularization and diabetic macular edema (DME) are two major clinicopathologic features during the development and progression of DR $[1,2]$. The occurrence of neovascularization is closely related to the duration of diabetes mellitus. Patients with recently diagnosed diabetes mellitus have a lower risk of proliferative diabetic retinopathy (PDR) involving neovascularization than those with longer duration [3]. DME is a major cause of visual impairment in DR patients, which can occur at any stage of DR, even at early and mild nonproliferative diabetic retinopathy (NPDR) stage [4]. Therefore, the prevention and early diagnosis of DME in NPDR are extremely important.

The pathogenesis of DME is multifactorial and remains unknown. Recent studies suggest that DME occurrence is induced by the breakdown of the blood-retina barrier (BRB) and the consequent increases in vascular permeability, vascular leakage, and fluid accumulation within the macula, which causes retina thickening, macular malfunction, 
and visual impairment [5]. Angiogenesis and inflammation play a critical role in the pathogenesis of DME involving many exudative cytokines [6-9].

As a potent angiogenesis factor, vascular endothelial growth factor (VEGF) can increase vascular permeability in DME pathogenesis [10]. At present, anti-VEGF therapy is a first-line treatment for DME. Studies have shown that VEGF inhibition effectively improved visual acuity and reduced macular thickness [11]. Nevertheless, the responses to anti-VEGF therapy were distinct in different cases. Specially, persistent DME did not improve even after several administrations of anti-VEGF drugs $[4,11]$. The above findings reveal that other mechanisms, independent of VEGF, may also contribute to DME.

However, previous studies mainly focused on inflammatory factors in DME, but not angiogenic factors. Furthermore, the conclusions from these studies were only based on the comparison of cytokine levels between DME and nondiabetic control $[7,11,12]$, which could not rule out the interference of diabetes and DR severities on cytokine level.

Here, we choose 25 angiogenic factors based on established and hypothesized angiogenesis pathway in DR and DME $[13,14]$, compared cytokines levels in the aqueous humor of NPDR patients with or without DME, and then investigated the effects of differentially expressed cytokines on optical coherence tomography angiography (OCTA) metrics to explore the potential molecular markers for DME in NPDR patients.

\section{Methods}

2.1. Study Subjects. This study included 21 eyes of 21 NPDR patients with DME (NPDR/DME+) who received intravitreal injection of anti-VEGF agents in the ophthalmology department of Xuzhou First People's Hospital from July 2017 to December 2018. 33 eyes of 33 senile cataract patients with diabetes mellitus who underwent phacoemulsification at the same time were enrolled, in which 17 eyes of 17 NPDR patients without DME (NPDR/DME-) and 16 eyes of 16 diabetic patients without retinopathy (DWR) were identified by slit-lamp biomicroscopy, fundus photography, and OCTA three days after the operation. DWR group served as controls. The inclusion criteria were as follows: (1) NPDR patients with DME, DME was defined with one or more of the follows: retinal thickening at or within $500 \mu \mathrm{m}$ of the macular center; hard exudates at or within $500 \mu \mathrm{m}$ of the macular center, also associated with adjacent retinal thickening; one or more zones of retinal thickening with one optic disc size, at least part of which within the range of one optic disc diameter in the macular center [15]. The diagnosis and classification of NPDR were based on the standards published by the international ophthalmological association [16]. (2) Senile cataract patients with type 2 diabetes mellitus who received phacoemulsification were diagnosed as NPDR without DME or DWR. Exclusion criteria were as follows: (1) proliferative diabetic retinopathy; (2) a history of vitreous hemorrhage, retinal detachment, intraocular surgery (except cataract surgery) or ocular trauma; (3) anti-VEGF or laser therapy previously; (4) complication with uveitis, glaucoma, optic nerve disease, or other eye diseases; (5) low signal strength index $(\mathrm{SSI}<50)$, blink artifacts or motion. This study followed the Declaration of Helsinki and was approved by the Ethics Committee of Xuzhou First People's Hospital (approval number: xyyll [2017] 008). Informed consent was obtained from all patients.

2.2. Ophthalmic Examination. All patients underwent comprehensive ophthalmic examination, including visual acuity, intraocular pressure, slit-lamp biomicroscopy, fundus photography, and OCTA. The images were diagnosed by two independent doctors, and cases with a discrepancy were reviewed by the third doctor with a higher qualification. OCTA (Optovue, Inc., Fremont, CA, USA) was performed using the angio retina mode. For each eye, a $3 \times 3 \mathrm{~mm}$ area centered on the fovea was scanned. Retinal thickness (RT) and retinal volume (RV) in the foveal and parafoveal area were automatically calculated by the built-in software from internal limiting membrane (ILM) to retinal pigment epithelium (RPE) layer. The fovea was defined as the circle area within the central $1 \mathrm{~mm}$ of the macula. Parafovea was defined as an area from the central $1 \mathrm{~mm}$ to the central $3 \mathrm{~mm}$ ring of the macular [17]. The OCTA images were automatically segmented to superficial capillary plexuses (SCP) and deep capillary plexuses (DCP) using the built-in software segmentation algorithm. The SCP was segmented with an inner boundary at $3 \mu \mathrm{m}$ beneath the ILM and an outer boundary at $15 \mu \mathrm{m}$ beneath the inner plexiform layer (IPL). The DCP was segmented with an inner boundary $15 \mu \mathrm{m}$ beneath the IPL and an outer boundary at $70 \mu \mathrm{m}$ beneath the IPL [18]. The vessel density values for the SCP and DCP in the whole, foveal, and parafoveal zones were calculated by the Angiovue Analytics built-in software. Vessel density was calculated as the percentage of pixels with flow signal above the preset decorrelation threshold in the defined region. FAZ area, perimeter, acircularity index, and FD-300 vessel density were automatically obtained via the FAZ assessment tool. FAZ surrounded by a continuous vascular closed ring was taken from ILM to outer plexiform layer (OPL). FD-300 was defined as a $300 \mu \mathrm{m}$ ring around the FAZ [17].

2.3. Sample Collection. Aqueous humor was collected before cataract surgery or intravitreal injection of anti-VEGF agents. After topical anesthesia, $100 \mu \mathrm{L}$ undiluted aqueous humor was withdrawn aseptically using an insulin syringe with a $30 \mathrm{G}$ needle at $1 \mathrm{~mm}$ inside the corneal limbus, which was placed in a $0.5 \mathrm{~mL}$ sterile Eppendorf tube and then stored at $-80^{\circ} \mathrm{C}$ until measurement.

2.4. Measurement of Cytokines. Twenty-five cytokines, including epidermal growth factor (EGF), hepatocyte growth factor (HGF), heparin-binding EGF-like growth factor (HBEGF), fibroblast growth factor 1 (FGF-1), FGF-2, FGF-19, FGF-21, FGF-23, granulocyte colony-stimulating factor (GCSF), bone morphogenetic protein 9 (BMP-9), Endoglin, Endothelin-1, Leptin, Follistatin, $\alpha$-Fetoprotein, FABP1, interleukin-8 (IL-8), Angiopoietin-2 (ANG-2), angiopoietinlike 3 (ANGPTL3), ANGPTL4, ANGPTL6, placental growth 
TABLE 1: Demographic characteristics of the 3 groups.

\begin{tabular}{|c|c|c|c|c|c|}
\hline Characteristic & $\operatorname{DWR}(N=16)$ & NPDR/DME- $(N=17)$ & $\mathrm{NPDR} / \mathrm{DME}+(N=21)$ & $F / \chi^{2}$ value & $P$ value (among the 3 groups) \\
\hline Age (yrs) & $67.94 \pm 8.80$ & $64.47 \pm 7.19$ & $64.76 \pm 11.61$ & 0.675 & $0.514^{\mathrm{a}}$ \\
\hline Male gender, no. (\%) & $5(31.25 \%)$ & $7(41.18 \%)$ & $14(66.67 \%)$ & 5.046 & $0.080^{\mathrm{b}}$ \\
\hline BMI & $24.80 \pm 3.16$ & $24.19 \pm 1.88$ & $25.97 \pm 3.27$ & 1.895 & $0.161^{\mathrm{a}}$ \\
\hline MAP & $100.33 \pm 7.41$ & $105.02 \pm 12.35$ & $101.95 \pm 11.07$ & 0.844 & $0.436^{\mathrm{a}}$ \\
\hline $\begin{array}{l}\text { Fasting plasma glucose } \\
(\mathrm{mmol} / \mathrm{l})\end{array}$ & $6.72 \pm 1.43$ & $7.42 \pm 1.91$ & $6.51 \pm 1.32$ & 1.667 & $0.199^{\mathrm{a}}$ \\
\hline HbAlc (\%) & $7.48 \pm 1.25$ & $7.97 \pm 1.48$ & $7.56 \pm 0.98$ & 0.789 & $0.460^{\mathrm{a}}$ \\
\hline Duration of diabetes (yrs) & $7.88 \pm 5.94$ & $11.88 \pm 4.96$ & $11.20 \pm 9.21$ & 1.485 & $0.236^{\mathrm{a}}$ \\
\hline NPDR classification & & & & 2.354 & $0.354^{\mathrm{b}}$ \\
\hline Mild (\%) & - & $5(29.41 \%)$ & $4(19.05 \%)$ & & \\
\hline Moderate (\%) & - & $8(47.06 \%)$ & $7(33.33 \%)$ & & \\
\hline Severe $(\%)$ & - & $4(23.53 \%)$ & $10(47.62 \%)$ & & \\
\hline
\end{tabular}

BMI: body mass index; MAP: mean arterial pressure; HbAlc: hemoglobin Alc; -: not analyzed. Values are mean \pm standard deviation unless otherwise indicated. ${ }^{\mathrm{a} O n e-w a y ~ a n a l y s i s ~ o f ~ v a r i a n c e ~ w i t h ~ p o s t ~ h o c ~ l e a s t ~ s i g n i f i c a n t ~ d i f f e r e n c e ~ m u l t i p l e ~ c o m p a r i s o n ~ t e s t s . ~}{ }^{\mathrm{b}} \mathrm{Chi}$-square test.

TABLE 2: Comparison of the cytokine levels in the aqueous humor among the 3 groups [M(Q1,Q3)].

\begin{tabular}{|c|c|c|c|c|c|}
\hline Aqueous cytokines & DWR $(\mathrm{pg} / \mathrm{ml})(N=16)$ & NPDR/DME- $(\mathrm{pg} / \mathrm{ml})(N=17)$ & $\mathrm{NPDR} / \mathrm{DME}+(\mathrm{pg} / \mathrm{ml})(N=21)$ & $\chi^{2}$ value & $P$ value \\
\hline HGF & $445.64(286.17,583.95)$ & $400.13(353.31,688.43)$ & $626.10(528.81,805.84)^{\mathrm{ab}}$ & 9.596 & 0.008 \\
\hline HB-EGF & $1.18(1.02,1.62)$ & $1.21(0.87,1.48)$ & $1.06(0.92,1.27)$ & 1.586 & 0.453 \\
\hline FGF-2 & $13.19(11.17,18.16)$ & $15.75(11.66,19.81)$ & $13.19(9.33,15.75)$ & 1.803 & 0.406 \\
\hline FGF-19 & $50.29(33.56,66.44)$ & $39.42(32.11,53.29)$ & $234.78(35.51,335.39)^{\mathrm{b}}$ & 8.362 & 0.015 \\
\hline Endothelin-1 & $8.15(6.24,13.59)$ & $8.83(5.97,10.64)$ & $8.22(5.78,10.23)$ & 0.763 & 0.683 \\
\hline Leptin & $68.33(61.73,80.97)$ & $64.84(57.66,79.31)$ & $88.26(68.33,135.53)$ & 5.895 & 0.052 \\
\hline IL-8 & $4.64(3.32,7.42)$ & $7.16(3.67,8.14)$ & $9.97(6.95,17.18)^{\mathrm{ab}}$ & 12.276 & 0.002 \\
\hline ANG-2 & $32.28(26.52,44.62)$ & $36.52(25.42,44.92)$ & $34.58(25.13,44.17)$ & 0.014 & 0.993 \\
\hline ANGPTL4 & $1529.50(1078.50,4347.25)$ & $2726.00(2025.00,12519.50)^{\mathrm{a}}$ & $23778.00(14490.00,26311.50)^{\mathrm{ab}}$ & 31.902 & $<0.001$ \\
\hline PLGF & $1.25(1.02,1.79)$ & $1.23(1.07,3.54)$ & $3.75(2.74,6.56)^{\mathrm{ab}}$ & 20.631 & $<0.001$ \\
\hline VEGF-A & $179.14(132.90,220.41)$ & $276.25(159.75,345.77)$ & $363.93(242.95,564.12)^{\mathrm{ab}}$ & 20.004 & $<0.001$ \\
\hline VEGF-C & $38.52(29.76,77.98)$ & $45.36(33.79,65.16)$ & $47.24(34.26,56.01)$ & 0.076 & 0.963 \\
\hline
\end{tabular}

HGF: hepatocyte growth factor; HB-EGF: heparin-binding EGF-like growth factor; FGF-2: fibroblast growth factor 2; FGF-19: fibroblast growth factor 19; IL8: interleukin 8; ANG-2: angiopoietin-2; ANGPTL4: angiopoietin-like 4; PLGF: placental growth factor; VEGF-A: vascular endothelial growth factor-A; VEGF-C: vascular endothelial growth factor-C. Cytokine levels $(\mathrm{pg} / \mathrm{mL})$ are presented as median with interquartile range. The levels of cytokines in the aqueous humor of the 3 groups were compared by Kruskal-Wallis $H$ test; one-to-one multiple comparisons were performed by Mann-Whitney $U$ test, a: compared with control, ${ }^{\mathrm{a}} P<0.0167$, b: compared with NPDR/ME- group, ${ }^{\mathrm{b}} P<0.0167$.

factor (PLGF), VEGF-A, VEGF-C, and VEGF-D (angiogenesis/growth factor panels, HAGP1MAG-12K and HLPPMAG-57K; Millipore Corporation, Billerica, MA, USA) were measured with Luminex bead-based multiplex array. All assays were performed strictly according to the manufacturer's guidelines, the detailed procedures of which were described in a previous study [19]. In brief, the assay buffer $(25 \mu \mathrm{L})$ was added to the background, standard, control, and sample wells. Each standard or control $(25 \mu \mathrm{L})$ was added into the appropriate wells. Aqueous humor $(25 \mu \mathrm{L})$ was added into the appropriate wells. Mixed beads $(25 \mu \mathrm{L})$ were added to each well. Seal, wrap with foil, and incubate with agitation on a plate shaker overnight at $4^{\circ} \mathrm{C}$. Gently remove fluid and wash plate 3 times. Next, detection antibody $(25 \mu \mathrm{L})$ was added, and the plate was incubated for 1 hour at room temperature. Then, streptavidin-
Phycoerythrin solution $(25 \mu \mathrm{L})$ was added to each well, and the plate was incubated for 30 minutes at room temperature. Gently remove well contents and wash plate 3 times. Sheath fluid $(100 \mu \mathrm{L})$ was added to all wells, and the beads were resuspended on a plate shaker for 5 minutes. Next, the plate was run on the FlexMAP 3D (Luminex) platform with xPONENT software. Median Fluorescent Intensity (MFI) was collected using Milliplex analyst 5.1 software. Standard curve for each cytokine was generated using the standards provided in the kits, and cytokine concentrations were obtained from the standard curves using a 5-parameter curve-fitting algorithm to transform the MFI values into concentrations.

2.5. Statistical Analyses. Statistical analysis was performed with SPSS 19.0. Shapiro-Wilk test was used to assess the 


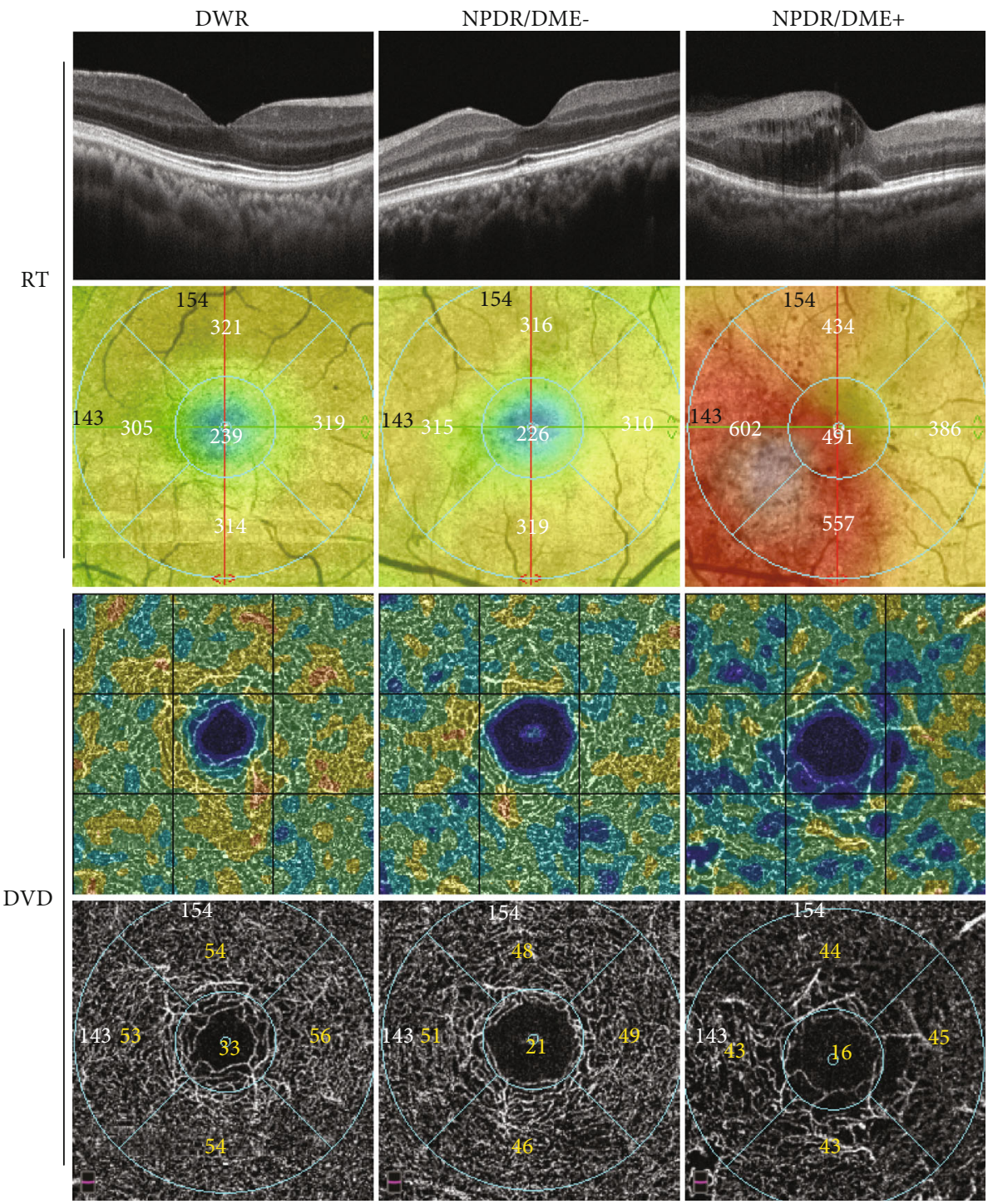

FIGURE 1: OCTA images of the three groups. Compared with DWR and NPDR/DME- groups, RT was significantly increased and DVD was significantly decreased in NPDR/DME+ groups.

normality of measurement variables. Normally distributed variables were expressed as mean \pm standard deviation, whereas skewed distributed variables were expressed as median (Q1, Q3). Categorical variables were summarized as counts and percentage. Comparisons of categorical variables were performed using chi-squared test. One-way analysis of variance with post hoc least significant difference (LSD) multiple comparison tests was performed for normally distributed variables among the three groups. Kruskal-Wallis $H$ test was performed for skewed variables among the three groups. Mann-Whitney $U$ test was performed for skewed variables between two groups, and a $P$ $<0.0167(0.05 / 3)$ was considered significant for multiple comparisons. Spearman's rank correlation test was performed to assess the association between cytokine levels and the OCTA metrics. The correlation coefficient was tested by Student's $t$ test and the cytokines with $P<0.05$ were included for single-factor linear regression analysis, and the cytokines with $P<0.05$ in the single factor linear regression were further included in the multiple linear regression model. RT (foveal and parafoveal), RV (foveal and parafoveal), and DVD (whole and parafoveal) were used as dependent variables, respectively, ANGPTL4 and VEGFA were used as independent variables, stepwise multiple linear regression was used to evaluate the cytokines that affect OCTA metrics, and $P<0.05$ was considered statistically significant.

\section{Results}

3.1. Demographic Characteristics. There were no significant differences in age, gender composition, body mass index (BMI), mean arterial pressure (MAP), fasting plasma glucose, $\mathrm{HbAlc}$, and the duration of diabetes among the three groups (all, $P>0.05$ ). There were no significant differences in severity degree of NPDR between NPDR/DME+ and NPDR/DME- groups $(P>0.05)$ (Table 1$)$. 
TABLE 3: Comparison of the OCTA Metrics among the 3 groups (mean $\pm \mathrm{SD}$ ).

\begin{tabular}{|c|c|c|c|c|c|}
\hline OCTA metrics & $\operatorname{DWR}(N=16)$ & NPDR/DME- $(N=17)$ & NPDR/DME $+(N=21)$ & $F$ value & $P$ value \\
\hline \multicolumn{6}{|l|}{$\mathrm{RT}(\mu \mathrm{m})$} \\
\hline Fovea & $240.90 \pm 20.57$ & $237.54 \pm 22.77$ & $331.76 \pm 90.87^{\mathrm{ab}}$ & 15.617 & $<0.001$ \\
\hline Parafovea & $308.59 \pm 13.68$ & $313.18 \pm 20.56$ & $379.25 \pm 76.61^{\mathrm{ab}}$ & 12.061 & $<0.001$ \\
\hline \multicolumn{6}{|l|}{$\mathrm{RV}\left(\mathrm{mm}^{3}\right)$} \\
\hline Fovea & $0.19 \pm 0.02$ & $0.19 \pm 0.02$ & $0.26 \pm 0.07^{\mathrm{ab}}$ & 15.640 & $<0.001$ \\
\hline Parafovea & $1.90 \pm 0.09$ & $1.93 \pm 0.14$ & $2.26 \pm 0.41^{\mathrm{ab}}$ & 9.838 & $<0.001$ \\
\hline \multicolumn{6}{|l|}{ SVD (\%) } \\
\hline Whole & $39.37 \pm 4.48$ & $40.20 \pm 3.63$ & $39.31 \pm 5.16$ & 0.211 & 0.810 \\
\hline Fovea & $12.24 \pm 5.28$ & $14.25 \pm 5.24$ & $15.04 \pm 7.04$ & 1.011 & 0.371 \\
\hline Parafovea & $42.35 \pm 4.86$ & $42.56 \pm 4.11$ & $41.01 \pm 5.80$ & 0.535 & 0.589 \\
\hline \multicolumn{6}{|l|}{ DVD (\%) } \\
\hline Whole & $49.05 \pm 3.38$ & $45.96 \pm 4.54$ & $42.35 \pm 5.66^{\mathrm{ab}}$ & 9.260 & $<0.001$ \\
\hline Fovea & $27.90 \pm 5.24$ & $25.08 \pm 6.20$ & $24.79 \pm 9.06$ & 0.962 & 0.389 \\
\hline Parafovea & $52.25 \pm 3.70$ & $48.64 \pm 5.19^{\mathrm{a}}$ & $44.76 \pm 5.94^{\mathrm{ab}}$ & 9.752 & $<0.001$ \\
\hline FD-300 vessel density (\%) & $43.57 \pm 5.00$ & $44.40 \pm 5.19$ & $42.54 \pm 5.91$ & 0.561 & 0.574 \\
\hline \multicolumn{6}{|l|}{ FAZ } \\
\hline Area $\left(\mathrm{mm}^{2}\right)$ & $0.34 \pm 0.08$ & $0.37 \pm 0.11$ & $0.42 \pm 0.25$ & 1.074 & 0.349 \\
\hline Perimeter $(\mathrm{mm})$ & $2.37 \pm 0.31$ & $2.57 \pm 0.42$ & $2.82 \pm 1.04$ & 1.871 & 0.164 \\
\hline Acircularity index & $1.16 \pm 0.05$ & $1.19 \pm 0.06$ & $1.23 \pm 0.12$ & 2.896 & 0.064 \\
\hline
\end{tabular}

SD: standard deviation; RT: retinal thickness; RV: retinal volume; SVD: superficial vessel density; DVD: deep vessel densities; FAZ: foveal avascular zone. Oneway ANOVA for normal distribution data among the 3 groups followed by post hoc least significant difference analysis between each two groups. a: compared with control; b: compared with NPDR/DME- group, $P<0.05$ was deemed to be statistically significant.

TABLE 4: Correlation between cytokine levels in aqueous humor and OCTA metrics in NPDR eyes $(n=38)$.

\begin{tabular}{lcccccccccccc}
\hline & \multicolumn{2}{c}{ Foveal RT } & \multicolumn{2}{c}{ Parafoveal RT } & \multicolumn{3}{c}{ Foveal RV } & \multicolumn{2}{c}{ Parafoveal RV } & \multicolumn{2}{c}{ Whole DVD } & \multicolumn{2}{c}{ Parafoveal DVD } \\
& $r_{s}$ & $P$ value & $r_{s}$ & $P$ value & $r_{s}$ & $P$ value & $r_{s}$ & $P$ value & $r_{s}$ & $P$ value & $r_{s}$ & $P$ value \\
\hline HGF & 0.250 & 0.130 & 0.211 & 0.203 & 0.249 & 0.132 & 0.127 & 0.447 & -0.087 & 0.605 & -0.144 & 0.389 \\
FGF19 & 0.315 & 0.054 & 0.332 & 0.042 & 0.314 & 0.055 & 0.297 & 0.070 & -0.256 & 0.121 & -0.293 & 0.074 \\
IL-8 & 0.175 & 0.293 & 0.158 & 0.345 & 0.172 & 0.302 & 0.041 & 0.807 & -0.171 & 0.304 & -0.184 & 0.268 \\
ANGPTL4 & 0.569 & $<0.001$ & 0.555 & $<0.001$ & 0.566 & $<0.001$ & 0.519 & 0.001 & -0.352 & 0.030 & -0.421 & 0.008 \\
PLGF & 0.363 & 0.025 & 0.352 & 0.030 & 0.365 & 0.024 & 0.326 & 0.046 & -0.240 & 0.147 & -0.288 & 0.080 \\
VEGF-A & 0.528 & 0.001 & 0.437 & 0.006 & 0.530 & 0.001 & 0.389 & 0.016 & -0.373 & 0.021 & -0.422 & 0.008 \\
\hline
\end{tabular}

RT: retinal thickness; RV: retinal volume; DVD: deep vessel density. Spearman test, $P<0.05$ was deemed to be statistically significant.

3.2. Comparison of Cytokine Levels in Aqueous Humor. The levels of ANGPTL4 among the three groups were significantly different from each other $(P<0.05)$. NPDR/DME+ group had the highest level of ANGPTL4 in the three groups, and NPDR/DME- group had a higher ANGPTL4 level than the DWR group (all, $P<0.0167$ ). For HGF, IL-8, PLGF, and VEGF-A, NPDR/DME+ group had the highest levels in the three groups (all, $P<0.0167$ ), but no significant difference between NPDR/DME- and DWR group was found (all, $P>0.0167$ ). In addition, NPDR/DME+ group had a higher level of FGF-19 than the NPDR/DME- group $(P<0.0167)$, but no significant changes were detected in NPDR/DME+ and NPDR/DME- groups, compared with the DWR group (all, $P>0.0167$ ). There were no significant differences of HB-EGF, FGF-2, Endothelin-1, Leptin, ANG-2, and VEGF-C among the three groups (all, $P>$ 0.05), and the levels of EGF, FGF-1, FGF-21, FGF-23, GCSF, BMP-9, Endoglin, Follistatin, AFP, FABP1, ANGPTL3, ANGPTL6, and VEGF-D were lower than the minimum detectable levels of the panel (Table 2).

3.3. Comparison of OCTA Metrics. Compared with DWR and NPDR/DME- group, RT and RV in NPDR/DME+ group were significantly increased, and the whole/parafoveal deep vessel densities (DVD) were reduced (Figure 1, Table 3). Besides, NPDR/DME- group had a lower parafoveal DVD than the DWR group (all, $P<0.05$ ) (Table 3 ). There were no significant differences of superficial vessel 
TABLE 5: Target cytokines affecting the OCTA Metrics in NPDR eyes $(n=38)$.

(a)

\begin{tabular}{|c|c|c|c|c|c|c|c|c|}
\hline \multirow{2}{*}{ Cytokines } & \multicolumn{4}{|c|}{ Foveal RT } & \multicolumn{4}{|c|}{ Parafoveal RT } \\
\hline & $\beta$ & SE & $P$ value & $R^{2}$ & $\beta$ & SE & $P$ value & $R^{2}$ \\
\hline ANGPTL4 & $4.299 \times 10^{-3}$ & $0.745 \times 10^{-3}$ & $<0.001$ & 0.480 & $3.598 \times 10^{-3}$ & $0.572 \times 10^{-3}$ & $<0.001$ & 0.523 \\
\hline VEGF-A & - & - & 0.050 & & - & - & 0.658 & \\
\hline
\end{tabular}

(b)

\begin{tabular}{|c|c|c|c|c|c|c|c|c|}
\hline \multirow{2}{*}{ Cytokines } & \multicolumn{4}{|c|}{ Foveal RV } & \multicolumn{4}{|c|}{ Parafoveal RV } \\
\hline & $\beta$ & SE & $P$ value & $R^{2}$ & $\beta$ & SE & $P$ value & $R^{2}$ \\
\hline ANGPTL4 & $3.371 \times 10^{-6}$ & $0.586 \times 10^{-6}$ & $<0.001$ & 0.479 & $17.705 \times 10^{-6}$ & $3.285 \times 10^{-6}$ & $<0.001$ & 0.447 \\
\hline VEGF-A & - & - & 0.050 & & - & - & 0.602 & \\
\hline
\end{tabular}

(c)

\begin{tabular}{lcccccrr}
\hline \multirow{2}{*}{ Cytokines } & \multicolumn{3}{c}{ Whole DVD } & P value & $R^{2}$ & \multicolumn{3}{c}{ Parafoveal DVD } \\
& $\beta$ & SE & $P$ vE & \multicolumn{2}{c}{$P$ value } & $R^{2}$ \\
\hline ANGPTL4 & $-1.705 \times 10^{-4}$ & $0.611 \times 10^{-4}$ & 0.008 & 0.178 & $-1.799 \times 10^{-4}$ & $0.665 \times 10^{-4}$ & 0.010 \\
VEGF-A & - & - & 0.148 & & - & -169 & 0.238 \\
\hline
\end{tabular}

RT: Retinal thickness; RV: retinal volume; DVD: deep vessel density; —: without data from statistics software, multivariate linear regression models, $P<0.05$ was deemed to be statistically significant.

density (SVD), foveal DVD, FD-300 vessel density, FAZ area, FAZ perimeter, and acircularity index among the three groups (all, $P>0.05$ ) (Table 3 ).

3.4. Correlation between Cytokine Levels in Aqueous Humor and OCTA Metrics. For all the NPDR patients, correlations between the above differentially expressed cytokines including HGF, FGF19, IL-8, ANGPTL4, PLGF, VEGF-A, and the OCTA metrics including RT, RV, and DVD were analyzed. The levels of ANGPTL4 and VEGF-A were positively correlated with foveal RT $(r s=0.569, P<0.001 ; r s=0.528, P=$ $0.001)$, parafoveal RT $(r s=0.555, P<0.001 ; r s=0.437, P=$ 0.006 ), foveal RV ( $r s=0.566, P<0.001 ; r s=0.530, P=0.001$ ), parafoveal RV ( $r s=0.519, P=0.001 ; r s=0.389, P=0.016$ ), and negatively correlated with whole DVD ( $r s=-0.352, P$ $=0.030 ; \quad r s=-0.373, \quad P=0.021)$ and parafoveal DVD ( $r s=-0.421, P=0.008 ; r s=-0.422, P=0.008)$. The level of PLGF was positively correlated with foveal RT ( $r s=0.363, P$ $=0.025)$, parafoveal RT $(r s=0.352, P=0.030)$, foveal RV ( $r s=0.365, P=0.024)$, and parafoveal RV ( $r s=0.326, P=$ $0.046)$ but not with DVD $(P>0.05)$. The level of FGF-19 was positively correlated with parafoveal RT ( $r s=0.332, P=$ 0.042 ) but not with foveal RT, RV, and DVD (all, $P>0.05$ ). There were no correlations between HGF, IL-8, and RT, RV, and DVD, respectively (all, $P>0.05$ ) (Table 4 ).

3.5. The Effect of Cytokines on OCTA Metrics. VEGF-A and ANGPTL 4 with $P<0.05$ in the single factor linear regression were further included in the multiple linear regression model (Table S1). Multiple regression analysis showed that the level of ANGPTL4 was an influencing factor for RT, RV, and DVD. The regression equations were fitted as follows: foveal $\mathrm{RT}=223.422+4.299 \times 10^{-3} \times$ ANGPTL4, parafoveal $\mathrm{RT}=$
$294.302+3.598 \times 10^{-3} \times$ ANGPTL4, foveal $\mathrm{RV}=0.176+$ $3.371 \times 10^{-6} \times$ ANGPTL4, parafoveal $\mathrm{RV}=1.838+17.705 \times$ $10^{-6} \times$ ANGPTL4, whole $\mathrm{DVD}=46.587-1.705 \times 10^{-4} \times$ ANGPTL4, and parafoveal DVD $=49.265-1.799 \times 10^{-4} \times$ ANGPTL4. Every additional $10^{3} \mathrm{pg} / \mathrm{ml}$ of ANGPTL4 was associated with an increase in foveal and parafoveal RT of $4.299 \mu \mathrm{m}$ and $3.598 \mu \mathrm{m}$, respectively. Every additional $10^{6} \mathrm{pg} / \mathrm{ml}$ of ANGPTL4 was associated with an increase in foveal and parafoveal RV of $3.371 \mathrm{~mm}^{3}$ and $17.705 \mathrm{~mm}^{3}$, respectively. Every additional $10^{4} \mathrm{pg} / \mathrm{ml}$ of ANGPTL4 was associated with a decrease in whole and parafoveal DVD of $1.705 \%$ and $1.799 \%$, respectively. The level of VEGF-A had no effect on RT, RV, and DVD (all, $P \geq 0.05$ ) (Table 5).

\section{Discussion}

Our study found that the levels of HGF, FGF-19, IL-8, ANGPTL4, PLGF, and VEGF-A increased in the aqueous humor of NPDR/DME+ patients, compared with NPDR/ DME- and DWR patients. Notably, the levels of VEGF-A and ANGPTL4 were correlated with RT, RV, and DVD, in which single factor linear regression showed that both VEGF-A and ANGPTL4 were the influencing factors for RT, RV, and DVD. These findings supported the previous reports that VEGF-A contributed to the pathogenesis of DME $[20,21]$. Cytokines with $P<0.05$ in the single factor regression were further included in the multiple linear regression model. Although the $P$ value for VEGF-A was 0.05 , we still considered VEGF-A as an influencing factor for macular edema in NPDR patients. Moreover, in the models with multiple cytokines, VEGF-A had a greater impact on foveal RT and RV than other OCTA metrics. 
Our study also found that the three groups were different from each other in the levels of ANGPTL4. Kwon et al. reported that both NPDR and PDR groups with similar severities of DME had higher levels of ANGPTL4 than the cataract controls, and the PDR group had a higher level than the NPDR group [22]. Here, we found that both DME+ and DME- groups with similar severities of NPDR had higher ANGPTL4 levels than the DWR group, and the DME+ group had a higher level than the DME- group. These results suggested that ANGPTL4 was also associated with of DME. Multiple regression analysis revealed that the level of ANGPTL4 in aqueous humor was an influencing factor for RT, RV, and DVD. NPDR patients with high levels of ANGPTL4 in the aqueous humor had higher foveal/parafoveal RT and RV and lower whole/parafoveal DVD than the patients with low levels of ANGPTL4.

As an angiogenesis factor, ANGPTL4 promotes the pathological processes of diverse eye diseases by enhancing angiogenesis, vascular permeability, and inflammation [23, 24]. Aqueous ANGPTL4 was obviously increased in PDR, inducing retinal neovascularization $[25,26]$. Lu et al. demonstrated that ANGPTL-4 regulated diabetic retinal inflammation and angiogenesis by, at least partly, activating profilin-1 both in human retinal microvascular endothelial cells and in diabetic rats. Moreover, the activation of the ANGPTL4 was dependent on the overexpression of its upstream regulatory factor, HIF-1 $\alpha$ [27]. Using the oxygen-induced retinopathy mouse model for ischemic retinopathy, Xin et al. provided the evidence that hypoxic Müller cells promoted vascular permeability by HIF-1dependent upregulation of ANGPTL4 [28]. They also observed that inhibition of ANGPTL4 expression reduced the angiogenic potential and vascular permeability of hypoxic retinal Müller cells, which was additive with inhibition of VEGF expression [26]. Further, they identified the ANGPTL4/NRP/RhoA pathway as a therapeutic target for DME [14]. In the present study, multiple regression analysis suggested that ANGPTL4 might be more sensitive for NPDR and DME than VEGF-A, as shown by increased RT, RV, and decreased DVD. Overall, we hypothesized that ANGPTL4 may participate in the pathogenesis of DME through the above pathways, and targeting both ANGPTL4 and VEGF may be essential for effective management of DME.

We evaluated macular perfusion using $3 \times 3 \mathrm{~mm}$ scan mode of OCTA which divided retina into foveal and parafoveal area. Compared with DWR and NPDR/DME- group, the whole and parafoveal DVD in NPDR/DME+ group were significantly decreased. The foveal DVD decreased with the severities of the three groups, although there was no statistical significance. Consistently, AttaAllah et al. [29] observed a reduction of parafoveal DVD in NPDR eyes with DME compared with diabetic eyes without DME. Furthermore, Toto et al. investigated the changes of retinal vessel in DME compared with normal controls and found a decrease in foveal and parafoveal DVD, especially in parafoveal area [30]. Similarly, we observed an obvious change of DVD in parafoveal area but not in foveal area, which may attribute to different controls included. Besides, Simonett et al. found that parafoveal DVD was decreased in DWR and NPDR patients compared with nondiabetic controls, while no difference was found in SVD [31, 32], which was consistent with our study. All these evidences suggested that, except for DR, the parafoveal nonperfusion in DCP was also an early indicator for DME. However, a large amount of data will be needed to determine the normal reference range of DVD.

Our study had several limitations. First, PDR patients without DME were usually treated with laser in clinical practice, and their aqueous humor samples could not be collected. For this reason, PDR patients were not included in our study. Further studies including PDR patients with or without DME are needed to explore the role of ANGPTL4 in all types of DR. Second, the stages of DME were not grouped, and thereby, ANGPTL4 involved in early or late stage of DME could not be determined. Considering the small sample size in this study, large sample size and detailed classification of DME will be necessary. Third, our study only analyzed the OCTA images with $3 \times 3 \mathrm{~mm}$ scan centered on the fovea, which could not reflect the blood flow of whole retina. Therefore, larger images should be analyzed in the future.

In conclusion, our study showed that microvascular change of NPDR patients with DME initially occurs at DCP with decreased vascular density in parafoveal area. The level of ANGPTL4 in aqueous humor was significantly increased in NPDR patients with DME, and ANGPTL4 was an influencing factor for RT, RV, and DCP, suggesting that ANGPTL4 may predict the progression of DME in NPDR patients.

\section{Data Availability}

The data used to support the findings of this study are available from the corresponding author upon request.

\section{Conflicts of Interest}

The authors declare that there is no conflict of interest regarding the publication of this paper.

\section{Acknowledgments}

This work was supported by Xuzhou science and technology project (social development key project) (grant number KC21153), Xuzhou Medical Innovation (Technical Breakthrough) Team from Xuzhou Health and Planning Committee (grant number XWCX201610), and Key Research and Development Program (Social Development) of Xuzhou (grant number KC20152).

\section{Supplementary Materials}

Supplemental Table 1: single factor linear regression between cytokines and OCTA metrics. Single-factor linear regression showed that ANGPTL4 and VEGF-A were the influencing factors of RT, RV, and DVD (all, $P<0.05$ ). (Supplementary Materials)

\section{References}

[1] T. Wang, J. Li, R. Xie et al., "Intraocular tumour necrosis factor ligand related molecule 1 A links disease progression of proliferative diabetic retinopathy after primary vitrectomy," Clinical 
and Experimental Pharmacology \& Physiology, vol. 47, no. 6, pp. 966-976, 2020.

[2] A. Markan, A. Agarwal, A. Arora, K. Bazgain, V. Rana, and V. Gupta, "Novel imaging biomarkers in diabetic retinopathy and diabetic macular edema," Therapeutic Advances in Ophthalmology, vol. 12, p. 251584142095051, 2020.

[3] D. P. Hainsworth, I. Bebu, L. P. Aiello et al., "Risk factors for retinopathy in type 1 diabetes: the DCCT/EDIC study," Diabetes Care, vol. 42, no. 5, pp. 875-882, 2019.

[4] S. Torres-Costa, M. C. Alves Valente, F. Falcao-Reis, and M. Falcao, "Cytokines and growth factors as predictors of response to medical treatment in diabetic macular edema," The Journal of Pharmacology and Experimental Therapeutics, vol. 373, no. 3, pp. 445-452, 2020.

[5] A. Daruich, A. Matet, A. Moulin et al., "Mechanisms of macular edema: beyond the surface," Progress in Retinal and Eye Research, vol. 63, pp. 20-68, 2018.

[6] F. Yenihayat, B. Ozkan, M. Kasap et al., "Vitreous IL-8 and VEGF levels in diabetic macular edema with or without subretinal fluid," International Ophthalmology, vol. 39, no. 4, pp. 821-828, 2019.

[7] M. Figueras-Roca, A. Sala-Puigdollers, S. Alforja et al., "Aqueous humour cytokine changes with intravitreal dexamethasone implant injection for diabetic macular edema," Ocular Immunology and Inflammation, vol. 27, no. 8, pp. 12031210, 2019.

[8] Y. R. Chung, Y. H. Kim, S. J. Ha et al., "Role of inflammation in classification of diabetic macular edema by optical coherence tomography," Journal Diabetes Research, vol. 2019, article 8164250, 8 pages, 2019.

[9] P. Romero-Aroca, M. Baget-Bernaldiz, A. Pareja-Rios, M. Lopez-Galvez, R. Navarro-Gil, and R. Verges, "Diabetic macular edema pathophysiology: vasogenic versus inflammatory," Journal Diabetes Research, vol. 2016, article 2156273, 17 pages, 2016.

[10] B. Sedziak-Marcinek, S. Teper, E. Chelmecka et al., "Diabetic macular edema treatment with bevacizumab does not depend on the retinal nonperfusion presence," Journal Diabetes Research, vol. 2021, article 6620122, 15 pages, 2021.

[11] Q. Wei, Z. Wan, Y. Hu, and Q. Peng, "Cytokine and chemokine profile changes in patients after intravitreal conbercept injection for diabetic macular edema," Drug Design, Development and Therapy, vol. 13, pp. 4367-4374, 2019.

[12] S. Bandyopadhyay, S. K. Bandyopadhyay, M. Saha, and A. Sinha, "Study of aqueous cytokines in patients with different patterns of diabetic macular edema based on optical coherence tomography," International Ophthalmology, vol. 38, no. 1, pp. 241-249, 2018.

[13] G. Wu, B. Liu, Q. Wu et al., "Correlations between different angiogenic and inflammatory factors in vitreous fluid of eyes with proliferative diabetic retinopathy," Frontiers in Medicine, vol. 8, article 727407, 2021.

[14] A. Sodhi, T. Ma, D. Menon et al., "Angiopoietin-like 4 binds neuropilins and cooperates with VEGF to induce diabetic macular edema," The Journal of Clinical Investigation, vol. 129, no. 11, pp. 4593-4608, 2019.

[15] M. B. Peres, R. T. Kato, V. F. Kniggendorf et al., "Comparison of optical coherence tomography angiography and fluorescein angiography for the identification of retinal vascular changes in eyes with diabetic macular edema," Ophthalmic Surgery, Lasers \& Imaging Retina, vol. 47, no. 11, pp. 1013-1019, 2016.
[16] C. P. Wilkinson, Ferris FL 3rd, R. E. Klein et al., "Proposed international clinical diabetic retinopathy and diabetic macular edema disease severity scales," Ophthalmology, vol. 110, no. 9, pp. 1677-1682, 2003.

[17] M. Inanc, K. Tekin, H. Kiziltoprak, S. Ozalkak, S. Doguizi, and Z. Aycan, "Changes in retinal microcirculation precede the clinical onset of diabetic retinopathy in children with type 1 diabetes mellitus," American Journal of Ophthalmology, vol. 207, pp. 37-44, 2019.

[18] K. Ghasemi Falavarjani, N. A. Iafe, J. P. Hubschman, I. Tsui, S. R. Sadda, and D. Sarraf, "Optical coherence tomography angiography analysis of the foveal avascular zone and macular vessel density after anti-VEGF therapy in eyes with diabetic macular edema and retinal vein occlusion," Investigative Ophthalmology \& Visual Science, vol. 58, no. 1, pp. 30-34, 2017.

[19] F. Zhang, P. Chang, Y. Zhao, and Y. Zhao, "A negative correlation of axial length with aqueous humor concentration of cytokines in patients with congenital cataracts," Molecular Vision, vol. 26, pp. 91-96, 2020.

[20] S. W. Lim, E. Bandala-Sanchez, M. Kolic et al., "The influence of intravitreal ranibizumab on inflammation-associated cytokine concentrations in eyes with diabetic macular edema," Investigative Ophthalmology \& Visual Science, vol. 59, no. 13, pp. 5382-5390, 2018.

[21] S. Y. Yu, D. H. Nam, and D. Y. Lee, "Changes in aqueous concentrations of various cytokines after intravitreal bevacizumab and subtenon triamcinolone injection for diabetic macular edema," Graefe's Archive for Clinical and Experimental Ophthalmology, vol. 256, no. 1, pp. 39-47, 2018.

[22] S. H. Kwon, J. P. Shin, I. T. Kim, and D. H. Park, “Aqueous levels of angiopoietin-like 4 and semaphorin 3E correlate with nonperfusion area and macular volume in diabetic retinopathy," Ophthalmology, vol. 122, no. 5, pp. 968-975, 2015.

[23] X. Yang, J. Cao, Y. Du, Q. Gong, Y. Cheng, and G. Su, “Angiopoietin-like protein 4 (ANGPTL4) induces retinal pigment epithelial barrier breakdown by activating signal transducer and activator of transcription 3 (STAT3): evidence from ARPE-19 cells under hypoxic condition and diabetic rats," Medical Science Monitor, vol. 25, pp. 6742-6754, 2019.

[24] X. Yang, Y. Cheng, and G. Su, "A review of the multifunctionality of angiopoietin-like 4 in eye disease," Bioscience Reports, vol. 38, no. 5, 2018.

[25] Q. Lu, W. Zou, B. Chen, C. Zou, M. Zhao, and Z. Zheng, "ANGPTL-4 correlates with vascular endothelial growth factor in patients with proliferative diabetic retinopathy," Graefe's Archive for Clinical and Experimental Ophthalmology, vol. 254, no. 7, pp. 1281-1288, 2016.

[26] S. Babapoor-Farrokhran, K. Jee, B. Puchner et al., "Angiopoietin-like 4 is a potent angiogenic factor and a novel therapeutic target for patients with proliferative diabetic retinopathy," Proceedings of the National Academy of Sciences of the United States of America, vol. 112, no. 23, pp. E3030-E3039, 2015.

[27] Q. Lu, P. Lu, W. Chen, L. Lu, and Z. Zheng, "ANGPTL-4 induces diabetic retinal inflammation by activating profilin1," Experimental Eye Research, vol. 166, pp. 140-150, 2018.

[28] X. Xin, M. Rodrigues, M. Umapathi et al., "Hypoxic retinal Muller cells promote vascular permeability by HIF-1dependent up-regulation of angiopoietin-like 4," Proceedings of the National Academy of Sciences of the United States of America, vol. 110, no. 36, pp. E3425-E3434, 2013.

[29] H. R. AttaAllah, A. A. M. Mohamed, and M. A. Ali, "Macular vessels density in diabetic retinopathy: quantitative assessment 
using optical coherence tomography angiography," International Ophthalmology, vol. 39, no. 8, pp. 1845-1859, 2019.

[30] L. Toto, R. D’Aloisio, M. di Nicola et al., "Qualitative and quantitative assessment of vascular changes in diabetic macular edema after dexamethasone implant using optical coherence tomography angiography," International Journal of Molecular Sciences, vol. 18, no. 6, p. 1181, 2017.

[31] J. M. Simonett, F. Scarinci, F. Picconi et al., "Early microvascular retinal changes in optical coherence tomography angiography in patients with type 1 diabetes mellitus," Acta Ophthalmologica, vol. 95, no. 8, pp. e751-e755, 2017.

[32] J. Khadamy, K. Abri Aghdam, and K. G. Falavarjani, "An update on optical coherence tomography angiography in diabetic retinopathy," J. Ophthalmic Vis. Res., vol. 13, no. 4, pp. 487-497, 2018. 